\title{
Water Resources Systems Analysis: A Bright Past and a Challenging but Promising Future
}

\author{
David E. Rosenberg, A.M.ASCE \\ Assistant Professor, Dept. of Civil and Environmental Engineering and \\ Utah Water Research Laboratory, Utah State Univ., 4110 Old Main, Logan, \\ UT 84322-4110. E-mail: david.rosenberg@usu.edu; and
}

\section{Kaveh Madani, A.M.ASCE}

Lecturer, Centre for Environmental Policy, Imperial College London, London SW7 2AZ, U.K.; formerly, Alex Alexander Assistant Professor, Dept. of Civil, Environmental and Construction Engineering, Univ. of Central Florida, 4000 Central Florida Blvd., Orlando, FL 32816. E-mail: k.madani@imperial.ac.uk

DOI: 10.1061/(ASCE)WR.1943-5452.0000414

The field of water resources systems analysis is now experiencing one of its most exciting eras where scientists, decision makers, and funding agencies want to apply systems approaches to solve varied, complex, uncertain, and interdisciplinary resource management problems. Solving these problems presents great opportunities for us to engage in complex, real-world decision-making and make positive changes. However, to capitalize on these opportunities, we as a field must also overcome several large challenges related to problem identification, integration, blind use of systems tools, a focus on optimality, and harnessing big data. To overcome, we must look back to find what we have accomplished, why we have sometimes failed, and how we can improve upon past work.

In May 2013, we had the privilege to organize and facilitate a thought-provoking panel discussion at the Environmental Water Resources Institute (EWRI) Congress in Cincinnati, Ohio, where different researcher and practitioner panelists spanning multiple generations plus esteemed audience members discussed the past and future of water resources systems analysis. Here, we distill some of the key points that emerged during the discussion that we think should guide our systems analysis work and research in the years and decades ahead.

\section{Apply and Use a Wide Range of Tools and Techniques}

Water resources systems analysts can make use of a wide range of tools and techniques to identify the relevant components of a system and study the interactions among those components. Since the Harvard Water Project and earlier, techniques like linear, nonlinear, multiobjective, and dynamic programming as well as evolutionary algorithms, multicriteria decision-making, and game theory have been widely applied to solve complex water problems in practically every region of the world (Harou et al. 2009; Labadie 1997; Madani 2010; Mirchi et al. 2010; Nicklow et al. 2010; Thiessen et al. 1998; Wurbs 2005; Yeh 1985). While some have criticized these techniques for failing to find actual use by decision makers (Rogers and Fiering 1986), the panelists noted that such assessments were based on a narrow review, only considered academic work, and ignored numerous industry applications like hydropower operations, scheduling, and planning where systems models predominate (Loucks et al. 1984). Although early work in the field focused on algorithm development, more recent efforts are tackling the increased complexity of problem formulation and computations using now larger computing capabilities. Today, we must make our analyses and tools more robust to include multiple objectives and decision makers, integrate more system components, identify larger promising solution sets, and use more readily available data.

\section{Develop the Science of Defining the Problem}

Our field can benefit greatly from new methods that further develop the science of defining problems - so that expert systems analysts can separately and reproducibly reach comparable problem definitions should they sit down to work on a common problem. Many of our pressing water problems are complex and wicked in that they are multifaceted, involve competing and often conflicting uses of water, and do not have clear technical or political solutions (Lund 2012). They require a systems approach to address. But what does a systems approach mean exactly in this context and what constitutes the system? What are the boundaries that define what we include and exclude from the analysis? These questions are necessarily open in that there are not observable physical data we can collect and apply to arrive at the single, correct problem definition. Problem definition may also be iterative (Lund 2008) and art as much as science.

Clear problem and system definitions allow others to both understand and reproduce the results that derive from the systems analysis work. Yet the need for a science to reproducibly define problems and systems may foster singular definitions and group-think that overlook important system components or interactions. Thus, we must simultaneously leave open opportunities to learn about the system-make learning endogenous-as we define the problem and subsequently model the system. Learning is often nonlinear and may require setbacks for later success. Setbacks may even be integral to ultimate success, and they certainly keep things interesting! And in overcoming setbacks, different people will likely learn different things about the system that result in different problem and system definitions that directly oppose the need for an objective, reproducible science of problem definition. Thus, we must balance the competing needs to clearly define our problems and systems, develop the science and methods of reproducible problem definition, and permit—even encourageopportunities to learn about the problems and systems on which we work. Further, our science of problem definition must accommodate, harmonize, and integrate multiple perspectives.

\section{Make Integration Central}

Our practice must include other branches of science such as ecology, biology, sociology, economics, policy, politics, the law, and others. We must consider the steady state as well as spatial and temporal dynamics and path dependencies. In short, we need to integrate all relevant system aspects, states, and metrics. Integration means we must also learn to effectively communicate with those 
who practice other disciplines: learn and adopt their language(s) as well as adapt our language so they can understand us.

We must also think how water interacts with food, energy, environment, politics, and other issues that are quite possibly of larger political importance. In effect, see water as part of a wider-potentially global-system and accordingly expand our boundaries of inquiry. Full integration requires us to think through and model the full set of feedbacks among systems. This more global perspective should guide us to identify strategies to manage water (and other resources) that sustain and enrich our environment for decades and centuries to come. Yet, we must also remember that we have limited capacities to understand and integrate; we cannot model everything. This limitation sets up a related and pressing question: how much integration and associated complexity is needed and required in our systems models?

\section{Start Small, Work Bigger, and Do Not Blindly Apply Model Tools}

As we contemplate large-scale integration in our systems models, we need to start small. First, develop simple models that represent key aspects of the system and provide useful insights to solve practical problems. Later, add complexity as needed. And above all else, avoid blindly adopting large, complex tools without properly framing the problem and thinking through the implications of the assumptions embedded into the models and tools we adopt.

Our expanding computational capabilities and associated capacity for large-scale integration allow us to confidently solve problems in fractions of seconds that are orders of magnitude larger and more complicated than problems our field's pioneers could ever dream to work on. We readily add model complexities as least publishable units with multiple objectives representing multiple decision criteria, stochasticity, uncertainties, new and faster solution algorithms, etc. Yet, model complexity does not necessarily correlate with usefulness. Although highly simplistic models can misguide policy (Madani 2013), super-complex models can also be misleading because we end up with black boxes that even the model developers cannot peer inside to understand how or why key model outputs and inputs are connected or correlated.

It is much harder to know what complexity is actually required to reach the overarching goals to learn about the problem at hand, solve the problem, and improve decision-making. Here, the keep it simple, stupid (KISS) approach can help: reduce complexity only to the level needed to develop an adequate understanding of the water resource system, and advise planners, managers, and decision makers on how to improve their system. Obviously, this level must be achievable with the available personnel, computer, and data resources. All of the preceding emphasize a parallel need for more work to demonstrate the use and impacts of new systems modeling methods by and on decision makers. In the years to come, we must balance the inherent tradeoffs between integration, complexity, available resources, understandability, and adoption as we integrate more features and components into our water resources systems models and draw on an expanding set of systems tools, methods, and models.

\section{Move Beyond Optimal}

We must move our systems analysis solution techniques beyond optimal to show decision makers the multiple good (or very good) solutions. For a long time, we have nearly exclusively focused on efficiently finding single optimal and Pareto-optimal solutions.
And for good reason, because optimization allows us to tractably weed out numerous poor-performing solutions in search of the single or Pareto best-performing one(s). Yet modeled optimal is often not optimal from the decision makers' points of view. The modeled and decision makers' objective(s) or constraints may differ. Or there are uncertainties (at the conceptual, formulation, and/or parameter levels) in how the model quantifies the objective(s) and/or constraints. Alternatively, decision makers may not be able to implement or sustain optimal or Pareto-optimal solutions prescribed by single- or multi-objective analysis because current models optimize system-wide and group objectives (such as aggregate net benefits) rather than individual objectives for individual stakeholders (Madani and Lund 2011). Two promising techniques that move beyond optimality include (1) near-optimal analysis, which identifies all the promising solutions that perform within a specified tolerance of the optimal solution (Brill et al. 1982; Rosenberg 2012); and (2) threshold detection to identify the range or points where changes in solutions matter (Brown et al. 2012). In addition, game theoretic, agent-based, and interactive multiobjective decision-making models and tools can further help identify solutions that are reachable, feasible, and stable-nearoptimal or Pareto-inferior solutions that decision makers may better accept and be more likely implement in practice (Read et al. 2014). Together, these techniques can identify new, promising solutions outside the optimality myopia.

\section{Harness Big Data}

We must also make use of recent advances in satellite, sensor, automation, networking, and computation capabilities to harness the ever-increasing avalanche of data and observations about the systems we study and use these data to build more accurate and integrated representations of our study systems. We are downstream users/consumers of data, as we rely on hydrologic, demand, infrastructure, system connectivity, downscaled climate, and other data to populate and run our models. Thus, as more data become available, we must understand where and how these data originate, choose which data to use, and automate the processes by which we search, discover, access, quality-check, transform, and input big data into our models. We must also get involved in discussions with data collectors and providers of what new data to collect, how to curate it, and provide access. These efforts will speed and ease the tasks of collecting and feeding data to our systems analysis efforts as well as electronically publishing model results. Simultaneously, the information contained within these data will likely change and transform the structure and content of our models. These capabilities will also soon allow us to push systems model results directly into the hands-literally_of decision makers and users.

\section{Wrap Up}

In the coming years and decades, our water resources systems analysis field holds great promise to help decision makers and researchers solve complex, uncertain, and interdisciplinary resource management problems. To do this, we must draw on a wide range of existing tools, develop the science of defining problems requiring a systems approach to solve, expand the conventional boundaries of water resource problems to include the views and expertise of other disciplines, move beyond identifying optimal and Pareto-optimal solutions, and better use available data. We must also start small, work to integrate more aspects of complex systems, and all the while leave ourselves opportunities to learn about the problems and systems 
we study as well as accommodate and integrate the varied lessons learned and new perspectives we acquire.

\section{Acknowledgments}

We thank panelists Pete Loucks, Avi Ostfeld, Pat Reed, and Emily Zechman Berglund as well as the attendees of the "Successes, State of the Art, and Future Challenges of Water Resources Systems Analysis" panel discussion session at the 2013 EWRI Congress for sharing their opinions and views that set the basis for writing this Editorial.

\section{References}

Brill, E. D., Jr., Chang, S.-Y., and Hopkins, L. D. (1982). "Modeling to generate alternatives: The HSJ approach and an illustration using a problem in land use planning." Manage. Sci., 28(3), 221-235.

Brown, C., Ghile, Y., Laverty, M. A., and Li, K. (2012). "Decision scaling: Linking bottom-up vulnerability analysis with climate projections in the water sector." Water Resour. Res., 48(9), W09537. .

Harou, J., Pulido-Velazquez, M., Rosenberg, D. E., Medellin-Azuara, J., Lund, J. R., and Howitt, R. E. (2009). "Hydro-economic models: Concepts, design, applications, and future prospects." J. Hydrol., 375(3-4), 627-643.

Labadie, J. W. (1997). "Reservoir system optimization models." Water Resour. Update, 108, 83-110.

Loucks, D. P., Stedinger, J. R., and Shamir, U. R. I. (1984). "Research in water resources and environmental systems modeling some historical perspectives, current issues, and future directions." Nat. Resour. Forum, 8(3), 219-240.

Lund, J. (2012). "Provoking more productive discussion of wicked problems." J. Water Resour. Plann. Manage., 10.1061/(ASCE)WR .1943-5452.0000190, 193-195.
Lund, J. R. (2008). Approaches to planning water resources, Univ. of California, Davis, Davis, CA, 19.

Madani, K. (2010). "Game theory and water resources." J. Hydrol., 381(3-4), 225-238.

Madani, K. (2013). "Modeling international climate change negotiations more responsibly: Can highly simplified game theory models provide reliable policy insights?" Ecol. Econ., 90, 68-76.

Madani, K., and Lund, J. R. (2011). "A Monte-Carlo game theoretic approach for multi-criteria decision making under uncertainty." Adv. Water Resour., 34(5), 607-616.

Mirchi, A., Watkins, D. Jr., and Madani, K. (2010). "Modeling for watershed planning, management, and decision making." Watersheds: Management, restoration and environmental impact, J. C. Vaughn, ed., Nova Science Publishers, New York.

Nicklow, J., et al. (2010). "State of the art for genetic algorithms and beyond in water resources planning and management." J. Water Resour Plann. Manage., 10.1061/(ASCE)WR.1943-5452.0000053, 412-432.

Read, L., Madani, K., and Inanloo, B. (2014). "Optimality versus stability in water resource allocation." J. Environ. Manage., 133, 343-354.

Rogers, P. P., and Fiering, M. B. (1986). "Use of systems analysis in water management." Water Resour. Res., 22(9), 146S-158S.

Rosenberg, D. E. (2012). "Near-optimal water management to improve multi-objective decision making." Int. Congress on Environmental Modelling and Software. Managing Resources of a Limited Planet: Pathways and Visions under Uncertainty, 6th Biennial Meeting, R. Seppelt, A. A. Voinov, S. Lange, and D. Bankamp, eds., International Environmental Modelling and Software Society (iEMSs), Leipzig, Germany, 104-111.

Thiessen, E. M., Loucks, D. P., and Stedinger, J. R. (1998). "Computerassisted negotiations of water resources conflicts." Group Decis. Negotiat., 7(2), 109-129.

Wurbs, R. A. (2005). "Comparative evaluation of generalized river/reservoir system models." TR-282, Texas Water Resources Institute, College Station, TX.

Yeh, W. W. G. (1985). "Reservoir management and operations models: A state of the art review." Water Resour. Res., 21(12), 1797-1818. 